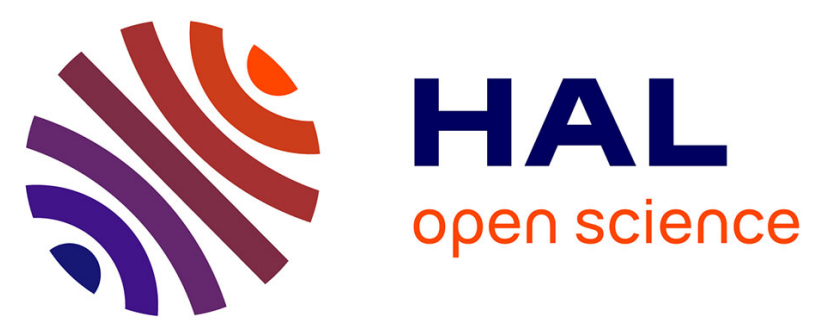

\title{
Continuous and discrete formulations for modeling electromagnetic wave propagation over an impedance ground in low troposphere in 3D
}

Hang Zhou, Alexandre Chabory, Rémi Douvenot

\section{To cite this version:}

Hang Zhou, Alexandre Chabory, Rémi Douvenot. Continuous and discrete formulations for modeling electromagnetic wave propagation over an impedance ground in low troposphere in 3D. APWC 2017 IEEE-APS Topical Conference on Antennas and Propagation in Wireless Communications, Sep 2017, Verone, Italy. pp. 181-184, ISBN: 978-1-5090-4453-5 10.1109/APWC.2017.8062274 . hal-01621273

\section{HAL Id: hal-01621273 \\ https://hal-enac.archives-ouvertes.fr/hal-01621273}

Submitted on 28 May 2018

HAL is a multi-disciplinary open access archive for the deposit and dissemination of scientific research documents, whether they are published or not. The documents may come from teaching and research institutions in France or abroad, or from public or private research centers.
L'archive ouverte pluridisciplinaire HAL, est destinée au dépôt et à la diffusion de documents scientifiques de niveau recherche, publiés ou non, émanant des établissements d'enseignement et de recherche français ou étrangers, des laboratoires publics ou privés. 


\title{
Continuous and Discrete Formulations for Modeling Electromagnetic Wave Propagation over an Impedance Ground in Low Troposphere in 3D
}

\author{
H. Zhou * A. Chabory * R. Douvenot *
}

\begin{abstract}
A 3D discrete mixed Fourier transform method is proposed for modeling the wave propagation over an impedance ground in $3 \mathrm{D}$. The continuous and discrete formulations of the propagators are presented. The discrete formulation achieves self-consistency with respect to the discrete electromagnetic theory. Numerical tests are performed to compare the two propagators. Both are shown to be accurate. Therefore, the self-consistent propagator should be preferred.
\end{abstract}

\section{INTRODUCTION}

Troposphere large-range propagation based on the parabolic equation method $(\mathrm{PE})$ has been an important research field for communications and radar [3]. The PE is an approximation of the wave equation valid along a paraxial direction neglecting the backward propagation. One of the most efficient methods for solving $\mathrm{PE}$ is the split-step Fourier (SSF) method [1]. It can consider any source, the geographical environment and realistic atmospheric conditions. The computation is performed going back and forth from a spatial to a spectral representation of the wave to evaluate the propagation iteratively. The corresponding spectral transform depends on the boundary conditions applied on the computation domain. Considering an impedance ground, the discrete mixed Fourier transform method (DMFT) [2] is used, which have firstly been introduced in $2 \mathrm{D}$. Then $3 \mathrm{D}-\mathrm{SSF}$ methods based on PE have been proposed [4]. However, a paraxial approximation is assumed to split the vertical and azimuthal derivative components.

In this paper, a 3D-SSF method based on the wave equation is proposed. This formulation is directly derived from the discretized wave equation to achieve self-consistency according to the discrete electromagnetic theory [6]. This work has already been performed for propagation over a PEC ground in [5]. The 2D spectral transform proposed here accounts for an impedance ground. Moreover, the propagators are extended to include the ground wave. Both the continuous (classical) and discrete

*Ecole Nationale de l'Aviation Civile, TELECOM-EMA, F-31055 Toulouse, France, Toulouse University, F-31400 Toulouse, France, e-mail: hang.zhou@recherche.enac.fr, douvenot@recherche.enac.fr. (self-consistent) formulations are presented. The propagation over an impedance ground with both propagators are tested and compared.

In section 2 , the configuration is introduced. In section 3 , the 3D-DMFT method is presented. In section 4 , the formulations of the continuous and discrete propagators are derived. In section 5 , numerical tests with two propagators are performed.

\section{Configuration}

The propagation is performed in the cylindrical coordinates $(r, \theta, z)$ with unit vectors $(\hat{\boldsymbol{r}}, \hat{\boldsymbol{\theta}}, \hat{\boldsymbol{z}})$. The wave sources are located into the cylinder $r \leq r_{0}$ and their radiated fields are known at $r=r_{0}$. The propagation is computed in the region $r>r_{0}, z \geqslant$ 0.

The computation domain is of finite size. The vertical domain is limited to $z \in\left[0, z_{\max }\right]$ and an impedance ground is assumed.

The fields are decomposed in one transverse electric (TE) and one transverse magnetic (TM) components oriented along $\hat{z}$. In this work, the TE component $\Psi$ is considered. The TM component can be formulated in a similar way.

\section{3D discrete mixed Fourier transform}

\subsection{General method}

For numerical reasons, a discretization is applied. The following uniform grid is used

$$
\begin{array}{lll}
r=r_{0}+p_{r} \Delta r & \text { for } & p_{r} \in\left\{1, \ldots, N_{r}\right\}, \\
z=p_{z} \Delta z & \text { for } & p_{z}=\left\{1, \ldots, N_{z}-1\right\}, \\
\theta=p_{\theta} \Delta \theta & \text { for } & p_{\theta}=\left\{0, \ldots, N_{\theta}-1\right\},
\end{array}
$$

with $\Delta r=\left(r_{\max }-r_{0}\right) / N_{r}, \Delta z=z_{\max } / N_{z}$ and $\Delta \theta=2 \pi / N_{\theta}$. This grid is shown in Fig. 1 .

The computation is performed marching on in distance. Between two consecutive cylinders, we propagate the field through a homogeneous medium. To account for refraction in the atmosphere, the phase screen method [2] is applied.

\subsection{Spectral transform}

The field $\Psi$ satisfies the $3 \mathrm{D}$ wave equation. On the azimuthal direction $\hat{\boldsymbol{\theta}}$, due to the $2 \pi$-periodic 


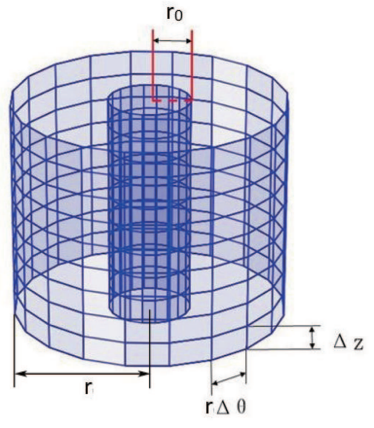

Figure 1: Grids along $\hat{\boldsymbol{z}}$ and $\hat{\boldsymbol{\theta}}$ on the initial cylinder at the distance $r_{0}$ and on a cylinder at $r>r_{0}$.

condition, the spectral transform amounts for a discrete Fourier transform (DFT). The spectrum of $\Psi$ corresponds to $\hat{\boldsymbol{\theta}}$ is denoted as $\bar{\Psi}$.

On the vertical direction $\hat{\boldsymbol{z}}$, the spectral representation of the DMFT is based on the finite-difference approximation of the Leontovich boundary condition [2] along the vertical axis $z$. We make a substitution from $\bar{\Psi}$ to $w$. For $p_{z}=\left\{1, \ldots, N_{z}-1\right\}$,

$$
w_{p_{r}, p_{z}, q_{\theta}}=\frac{\bar{\Psi}_{p_{r}, p_{z}+1, q_{\theta}}-\bar{\Psi}_{p_{r}, p_{z}-1, q_{\theta}}}{2 \Delta z}+\alpha \bar{\Psi}_{p_{r}, p_{z}, q_{\theta}},
$$

where

$$
\begin{aligned}
& w_{p_{r}, p_{z}, q_{\theta}}=w\left(r_{0}+p_{r} \Delta r, p_{z} \Delta z, q_{\theta}\right), \\
& \bar{\Psi}_{p_{r}, p_{z}, q_{\theta}}=\bar{\Psi}\left(r_{0}+p_{r} \Delta r, p_{z} \Delta z, q_{\theta}\right),
\end{aligned}
$$

and $\alpha$ is an impedance coefficient [2]. The spectrum $W_{p_{r}, q_{z}, q_{\theta}}$ is given by

$$
\begin{aligned}
W_{p_{r}, 0, q_{\theta}} & =A \sum_{p_{z}=0}^{N_{z}}{ }^{\prime} R^{p_{z}} \bar{\Psi}_{p_{r}, q_{z}, q_{\theta},} \\
W_{p_{r}, N_{z}, q_{\theta}} & =A \sum_{p_{z}=0}^{N_{z}}{ }^{\prime}\left(-\frac{1}{R}\right)^{p_{z}} \bar{\Psi}_{p_{r}, q_{z}, q_{\theta}}, \\
W_{p_{r}, q_{z}, q_{\theta}} & =\sum_{p_{z}=1}^{N_{z}-1} w_{p_{r}, q_{z}, q_{\theta}} \sin \left(\frac{\pi q_{z} p_{z}}{N}\right), \\
\text { with } \quad q_{z} & =\left\{1, \ldots, N_{z}-1\right\},
\end{aligned}
$$

where $R$ satisfies $R^{2}+2 \alpha \Delta z R-1=0$, and $A=$ $\frac{2\left(1-R^{2}\right)}{\left(1+R^{2}\right)\left(1-R^{\left.2 N_{z}\right)}\right.}$. Besides, the prime superscript on the sum indicates that the first and last terms are weighted with a coefficient $1 / 2$.

The propagation from $r$ to $r+\Delta r$ is computed by multiplying $M$ by a diagonal operator, denoted as the propagator. Two formulations of the propagators are presented in the next section.

Then the inverse transform is performed. First, $\bar{\Psi}_{p_{r}+1, p_{z}, q_{\theta}}$ is retrieved from $W_{p_{r}+1, q_{z}, q_{\theta}}$ as described for the 2D DMFT algorithm [2]. Then, an inverse DFT on $\hat{\boldsymbol{\theta}}$ is performed to obtain $\Psi_{p_{r}+1, p_{z}, p_{\theta}}$.

\section{Propagators}

The propagators are derived from the solution of the spectral wave equation. Two formulations are presented here. The first is the continuous formulation, which is a natural extension of the DMFT method [2] in 3D. We have highlighted an inconsistency of 2D-DMFT in [7]. The inconsistency problem of the 3D extension will be presented here. Then, a discrete formulation is proposed to achieve self-consistency.

\subsection{Continuous formulation}

The field $\Psi$ satisfies the $3 \mathrm{D}$ wave equation:

$\frac{\partial^{2} \Psi}{\partial r^{2}}+\frac{1}{r^{2}} \frac{\partial^{2} \Psi}{\partial \theta^{2}}+\frac{\partial^{2} \Psi}{\partial z^{2}}+\left(k_{0}^{2} n^{2}+\frac{1}{4 r^{2}}\right) \Psi=0$.

As introduced in the previous section, the discrete spectral transform amounts to a DFT along $\hat{\boldsymbol{\theta}}$ and a DMFT along $\hat{\boldsymbol{z}}$. However, in the continuous formulation, the spectral component is cast as a continuous function. Thus, the continuous spectral representation of wave equation is obtained.

From the solution of this spectral equation, the propagator is derived: for $q_{z}=\left\{1, \ldots, N_{z}-1\right\}$,

$$
W_{p_{r}+1, q_{z}, q_{\theta}}=\frac{H_{\kappa}^{(2)}\left(k_{r} r_{2}\right)}{H_{\kappa}^{(2)}\left(k_{r} r_{1}\right)} \sqrt{\frac{r_{2}}{r_{1}}} W_{p_{r}, q_{z}, q_{\theta}},
$$

where $r_{1}=r_{0}+p_{r} \Delta r, r_{2}=r_{0}+\left(p_{r}+1\right) \Delta r$, and $H_{\kappa}^{(2)}$ denotes the Hankel function of the second kind of order $\kappa$ with $\kappa=q_{\theta}, q_{\theta}=\left\{0, \ldots, N_{\theta}-1\right\}$.

Besides, $k_{r}^{2}=k_{0}^{2} n^{2}-k_{z}^{2}$ with

$$
k_{z}=q_{z} \pi / z_{\max } .
$$

The ground and sky waves are propagated by substituting $W_{p_{r}, 0, q_{\theta}} R \frac{z}{\Delta z}$ and $W_{p_{r}, N_{z}, q_{\theta}}\left(-\frac{1}{R}\right)^{\frac{z}{\Delta z}}$ into the wave equation and solving. This yields

$$
\begin{aligned}
W_{p_{r}, 0, q_{\theta}} & =\frac{H_{\kappa}^{(2)}\left(k_{s w_{1}} r_{2}\right)}{H_{\kappa}^{(2)}\left(k_{s w_{1}} r_{1}\right)} \sqrt{\frac{r_{2}}{r_{1}}} W_{p_{r}, 0, q_{\theta}}, \\
W_{p_{r}, N_{z}, q_{\theta}} & =\frac{H_{\kappa}^{(2)}\left(k_{s w_{2}} r_{2}\right)}{H_{\kappa}^{(2)}\left(k_{s w_{2}} r_{1}\right)} \sqrt{\frac{r_{2}}{r_{1}}} W_{p_{r}, N_{z}, q_{\theta}},
\end{aligned}
$$

with $k_{s w_{1}}=\sqrt{k^{2}+(\ln R)^{2} /(\Delta z)^{2}}$ and $k_{s w_{2}}=$ $\sqrt{k^{2}+(\ln (-R))^{2} /(\Delta z)^{2}}$. Note here, the sky wave is not considered in practical since apodization layer is applied on the top half domain.

This method is based on a finite-difference approximation for the ground boundary condition, its propagator in the spectral domain is obtained from the continuous spectral equations. To overcome this inconsistency, we propose a discrete formulation in the following subsection. 


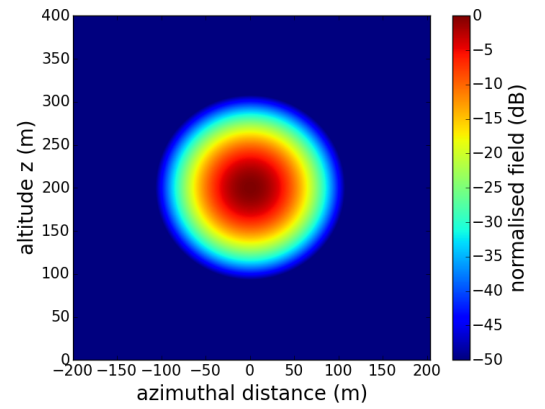

Figure 2: Normalised final electric field of the analytic solution of a CSP in 3D

\subsection{Discrete formulation}

To render our spectral representation numerically self-consistent, discretization is applied a prior$i$. Central-difference approximations substitute the derivatives along $\hat{\boldsymbol{\theta}}$ and $\hat{\boldsymbol{z}}$ in (5). Then the discrete spectral transforms are applied. The propagators are derived from the discrete spectral wave equation. The derivations are similar as in the continuous case.

The ground and sky waves are propagated by substituting $W_{p_{r}, 0, q_{\theta}} R^{p_{z}}$ and $W_{p_{r}, N_{z}, q_{\theta}}\left(-\frac{1}{R}\right)^{p_{z}}$ into the wave equation and solving. The propagator is the same as in (6) except for $\kappa$ and $k_{z}$, which are now given by

$$
\begin{aligned}
\kappa & =\frac{2}{\Delta \theta} \sin \left(\frac{\pi q_{\theta}}{N_{\theta}}\right) \text { with } q_{\theta}=\left\{0, \ldots, N_{\theta}-1\right\}, \\
k_{z} & =\frac{2}{\Delta z} \sin \left(\frac{\pi q_{z}}{2 N_{z}}\right) \text { with } q_{z}=\left\{1, \ldots, N_{z}-1\right\} .
\end{aligned}
$$

The propagation of surface waves is the same as (8) with $k_{s w_{1}}=\sqrt{k^{2}+\left(R+R^{-1}-2\right) /(\Delta z)^{2}}$ and $k_{s w_{2}}=\sqrt{k^{2}+\left((-R)+(-R)^{-1}-2\right) /(\Delta z)^{2}}$.

\section{Numerical tests}

\subsection{D propagation in free space}

In order to compare the propagation with the continuous and discrete propagators, a complex source point (CSP) [8] is propagated with both propagators. The results are compared to its analytic expression.

The frequency is $3 \mathrm{GHz}$. The complex source is located at $r_{s}=r_{s r}+\frac{j k_{0} W_{0}^{2}}{2}, z_{s}=200 \mathrm{~m}$, with the beam's width $W_{0}=3 \mathrm{~m}$ and $r_{s r}=800 \mathrm{~m}$.

The simulation parameters are: $r_{0}=1 \mathrm{~km}$, $r_{\max }=5 \mathrm{~km}, \Delta r=400 \mathrm{~m}, z_{\max }=200 \mathrm{~m}$, $\Delta z=0.1 \mathrm{~m}$, and $N_{\theta}=60000$. The choices of $\Delta z$ and $N_{\theta}$ satisfy the Nyquist criteria.

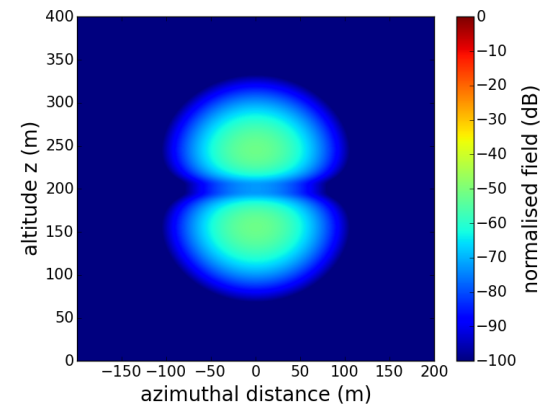

(a) Difference continuous SSF - analytic solution

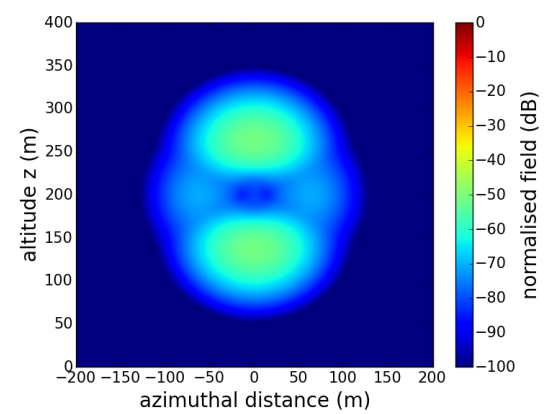

(b) Difference discrete SSF - analytic solution

Figure 3: Propagation of a 3D CSP in free-space.

The 3D propagation in free-space is performed. The final field of the analytic solution is plotted in Fig. 2 as a reference. The difference of SSF methods with the continuous and discrete propagators to the analytic solution are plotted in Fig. 3. The maximum difference between the continuous SSF and the analytic solution $D i f_{c}=-51.7 \mathrm{~dB}$. And the one of the discrete case $D i f_{d}=-51.0 \mathrm{~dB}$. Both cases are accurate for free-space propagation.

If we double the discretization step on $\hat{\boldsymbol{z}}$ and $\hat{\boldsymbol{\theta}}$ (i.e., $\Delta z=0.2 \mathrm{~m}, N_{\theta}=30000$ ), the differences become Dif $f_{c}=-51.8 \mathrm{~dB}$ and Dif $_{d}=-37.0 \mathrm{~dB}$. The discrete case is a little less accurate, since a FD approximation is applied.

\subsection{D propagation over an impedance ground}

The propagation over an impedance ground is considered with a relative permittivity $\epsilon_{r}=20$ and a conductivity $\sigma=0.02 \mathrm{~S} / \mathrm{m}$.

A complex source point is considered with $z_{s}=$ $15 \mathrm{~m}$. The other parameters are the same as in the previous test. An apodization is applied on the upper half domain.

The final field using the geometric optical (GO) method is plotted in Fig. 4 as a reference. The differences of SSF with the two propagators to GO 


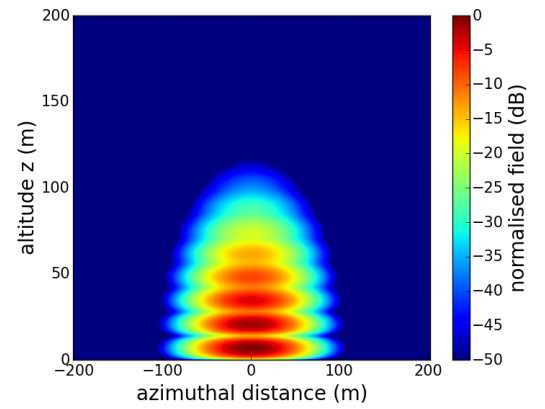

Figure 4: Normalised final electric field of GO for a propagation over an impedance ground

are plotted in Fig. 5. The maximum difference of the continuous case is $-52.4 \mathrm{~dB}$ and the one of the discrete case is $-51.9 \mathrm{~dB}$. The 3D-DMFT method with both formulations are accurate.

\section{Conclusion}

In this work, the aim is to simulate the $3 \mathrm{D}$ propagation over an impedance ground. The $2 \mathrm{D}$ discrete mixed Fourier transform method (DMFT) has been extended to 3D. Then the continuous and discrete formulations for the propagator have been presented. The continuous formulation is a natural extension of 2D-DMFT. However, an inconsistency of this method has been highlighted. Then, the discrete formulation has been proposed to achieve selfconsistency in the discrete domain. The simulation accuracy of 3D-DMFT with both propagators have been compared in the numerical tests. An analytic solution of a complex source point has been used as a reference. Propagations in free space and over an impedance ground are performed. The results of 3D-DMFT with both propagators are accurate. This method is successfully tested. The discrete formulation achieves a self-consistency. Therefore, the latter should be preferred for numerical simulations.

\section{References}

[1] R. H. Hardin, F. D. Tappert, "Applications of the split-step Fourier method to the numerical solution of nonlinear and variable coefficient wave equations", Siam Rev, vol. 15, no. 2, pp. 423, 1973

[2] G. D. Dockery, J. R. Kuttler, "An improved impedance-boundary algorithm for Fourier split-step solutions of the parabolic wave equation", IEEE Trans. Antennas Propag., vol. 44, no. 12 , pp.1592-1599, 1996

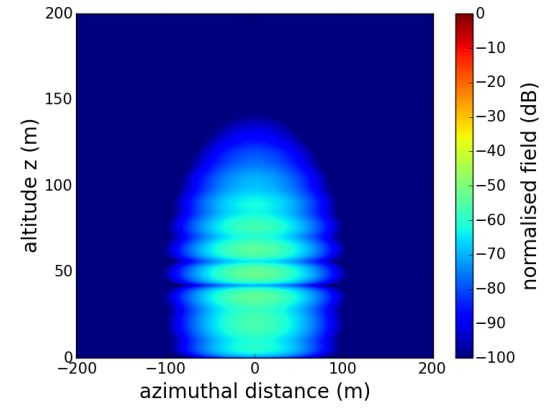

(a) Difference continuous SSF - GO

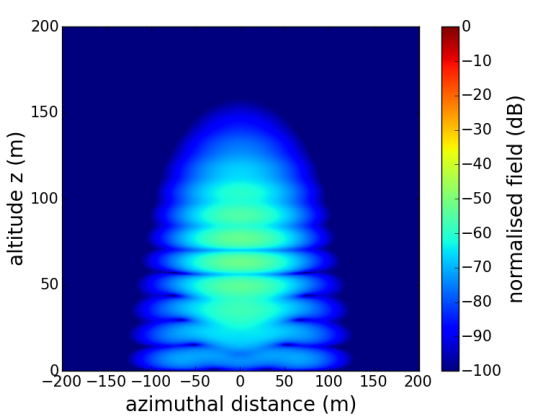

(b) Difference discrete SSF - GO

Figure 5: Propagation of a 3D complex source point over an impedance ground.

[3] M. Levy, Parabolic Equation Methods for Electromagnetic Wave Propagation, ser. IEE electromagnetic waves series 45, IET, Ed., 2000

[4] W. L. Siegmann, G. A. Kriegsmann, and D. Lee, "A wide-angle three-dimensional parabolic wave equation", J. Acoust. Soc. Am., vol. 78, no. 2, pp. 659-664, 1985

[5] H. Zhou, A. Chabory, and R. Douvenot, "A 3 D split-step Fourier algorithm based on a discrete spectral representation of the propagation equation", IEEE Trans. Antennas Propag., vol. 65, no. 4, pp. 1988-1995, 2017

[6] W. C. Chew, "Electromagnetic theory on a lattrice", J. Appl. Phys., vol. 75, no. 10, pp. 48434850, 1994

[7] H. Zhou, A. Chabory, and R. Douvenot, "Comparisons of discrete and continuous propagators for the modeling of low tropospheric propagation", 11th European Conference on Antennas and Propagation (EUCAP), Paris, 2017

[8] G. A. Deschamps, "Gaussian beam as a bundle of complex rays", Electronics letters, vol. 7, no. 23, pp. 684-685, 1971 